\title{
Efecto de la estación sobre las características seminales de alpacas (Vicugna pacos) criadas a nivel del mar
}

\author{
SEASONAL EFFECT ON THE SEMINAL CHARACTERISTICS OF ALPACAS (Vicugna pacos) \\ RAISED AT SEA LEVEL \\ Juan C. Villanueva M. ${ }^{1}$, Willian F. Huanca M. ${ }^{1}$, Fred Hilari O. ${ }^{1}$, Melania Uchuari P. ${ }^{1}$, \\ Francisco Rodríguez G. ${ }^{2}$, Wilfredo Huanca L., ${ }^{1,3}$
}

\section{Resumen}

El objetivo del presente estudio fue evaluar el efecto de la estación (verano, invierno) sobre las variables seminales de alpacas criadas bajo condiciones de la costa peruana. El estudio se realizó entre los meses de enero a setiembre de 2015 en el departamento de Lima. Se colectaron muestras de semen de 4 alpacas machos de 6-7 años, alimentadas con heno de alfalfa. Se realizaron cinco colecciones, mediante vagina artificial, por animal en cada estación: verano (enero-marzo) e invierno (julio-septiembre) con intervalos de 5 días entre colección (20 colecciones por estación). Se evaluaron las características macroscópicas (volumen, $\mathrm{pH}$, viscosidad, color) y microscópicas (concentración, motilidad, vitalidad, porcentaje de anormalidades, anormalidades de cola y cabeza, gota citoplasmática proximal y distal, y test de endosmosis). Los resultados obtenidos en el invierno presentan diferencias significativas $(\mathrm{p}<0.05)$ para las variables de concentración $\left(135.9 \pm 88.7 \times 10^{6} / \mathrm{ml}\right.$ vs $\left.242.4 \pm 140.9 \times 10^{6} / \mathrm{ml}\right)$, motilidad $(58.0 \pm 22.1 \%$ vs $77.3 \pm$ $11.6 \%)$, vitalidad $(52.9 \pm 18.4 \%$ vs $64.5 \pm 15.9 \%)$, viscosidad $(5.9 \pm 3.4 \mathrm{~cm}$ vs $3.7 \pm 2.0 \mathrm{~cm})$ y frecuencia de espermatozoides normales $(82.6 \pm 7.2 \%$ vs $88.1 \pm 7.0 \%)$ para verano e invierno, respectivamente. Los resultados obtenidos indican que la calidad de semen de alpacas, bajo las condiciones donde se realizó el estudio, difiere entre estaciones.

Palabras clave: alpaca; semen; estación

\section{Abstract}

The objective of the present study was to evaluate the effect of the season (summer, winter) on the seminal variables of alpacas reared under conditions of the Peruvian coast. The study was conducted between January to September 2015 in Lima, Peru. Semen

\footnotetext{
${ }^{1}$ Laboratorio de Reproducción Animal, Facultad de Medicina Veterinaria, Universidad Nacional Mayor de San Marcos, Lima, Perú

${ }^{2}$ Laboratorio Central, Universidad Alas Peruanas, Lima, Perú

${ }^{3}$ E-mail: whuanca2002@yahoo.com

Recibido: 7 de agosto de 2017

Aceptado para publicación: 28 de diciembre de 2017
} 
samples were collected from four 6-7 year-old male alpacas, fed alfalfa hay. Five collections per animal were made, using artificial vagina, in each season: summer (January-March) and winter (July-September) with intervals of 5 days between collections ( 20 collections per season). Macroscopic characteristics (volume, $\mathrm{pH}$, viscosity, color) and microscopic characteristics (concentration, motility, vitality, percentage of abnormalities, tail and head abnormalities, proximal and distal cytoplasmic droplet, and endosmosis test) were evaluated. The results obtained in the winter showed significant differences $(\mathrm{p}<0.05)$ for concentration $\left(135.9 \pm 88.7 \times 10^{6} / \mathrm{ml}\right.$ vs $\left.242.4 \pm 140.9 \times 10^{6} / \mathrm{ml}\right)$, motility $(58.0 \pm 22.1 \%$ vs 77.3 $\pm 11.6 \%)$, vitality $(52.9 \pm 18.4 \%$ vs $64.5 \pm 15.9 \%)$, viscosity $(5.9 \pm 3.4 \mathrm{~cm}$ vs $3.7 \pm 2.0 \mathrm{~cm})$ and normal sperm frequency $(82.6 \pm 7.2 \%$ vs $88.1 \pm 7.0 \%)$ for summer and winter, respectively. The results obtained indicate that the semen quality of alpacas, under the conditions of the study, differs between seasons.

Key words: alpaca; semen; season

\section{INTRODUCCIÓN}

Los camélidos sudamericanos (CSA) habitan los ecosistemas alto-andinos, generalmente sobre los $3800 \mathrm{msnm}$, en condiciones medio ambientales difíciles y con pasto de mala calidad; sin embargo los CSA han podido adaptarse y producir fibra de gran calidad y carne (Fernández-Baca, 1991; FAO, 1996).

En las crianzas de toda especie animal, el macho cumple un rol importante, tanto en el proceso reproductivo como en el mejoramiento genético, de allí que de su correcta selección y los cuidados que se le prodiguen dependerá el éxito de la explotación (Fernández-Baca, 1991). Los CSA presentan características reproductivas propias, incluyendo el semen; el cual, por su alta viscosidad, representa un problema para su manejo bajo las condiciones de laboratorio. No obstante, esta característica protege a los espermatozoides en el tracto reproductivo de la hembra (Troedsson et al., 2005).

La temperatura testicular normal en los rumiantes se mantiene entre 2 y $8{ }^{\circ} \mathrm{C}$ por debajo de la temperatura corporal (Ungerfeld, 2002). Por otro lado, el efecto de temperaturas altas sobre los testículos afecta la espermatogénesis por el efecto del estrés oxidativo, especialmente a los espermatocitos y las espermátides (Pérez-Crespo et al., 2008).

En un trabajo realizado con alpacas sobre los 3800 msnm en Puno, Perú, se señala que la estación (época seca y época de lluvia) afecta ciertas características del semen (Huanca et al., 2011), mientras que en un trabajo similar en llamas (Lama glama) en Argentina se obtuvo mayor concentración de espermatozoides en invierno que en verano, con menos anormalidades en la cola de espermatozoides (Giuliano et al., 2008).

\section{Materiales y Métodos}

\section{Ubicación del Estudio}

El presente trabajo se realizó entre los meses de enero a setiembre de 2015 en las instalaciones del Laboratorio de Reproducción Animal de la Facultad de Medicina Veterinaria, Universidad Nacional Mayor de San Marcos, ubicada en San Borja, Lima (Perú). Esta área se encuentra en la costa, a nivel del mar.

La temperatura ambiental en la época de verano tuvo una media de $24.2 \pm 2.9^{\circ} \mathrm{C}$ con temperatura máxima de 30.0 y mínima 
de $19.5^{\circ} \mathrm{C}$, mientras que en la época de invierno tuvo una media de $18.3 \pm 0.9^{\circ} \mathrm{C}$ con temperaturas máximas de 24 y mínimas de $15.2{ }^{\circ} \mathrm{C}$.

\section{Colección y Evaluación de Semen}

La muestra estuvo conformada por 40 eyaculados de cuatro alpacas de 6 a 7 años. Se obtuvieron cinco colecciones de semen por animal en cada estación: verano (eneromarzo) e invierno (julio-septiembre). Los animales se encontraban en buenas condiciones de salud y eran alimentados a base de alfalfa y agua ad libitum.

El semen se colectó mediante vagina artificial (VA) desarrollada por Sumar y Leyva (1981) y modificada por Huanca y Gauly (2001), con la ayuda de una hembra receptiva. Los eyaculados fueron colocados inmediatamente en estufa a $37^{\circ} \mathrm{C}$. Asimismo, el material de laboratorio que podría entrar en contacto con el semen se mantuvo a $37^{\circ} \mathrm{C}$ para evitar el shock térmico.

Se hicieron las siguientes determinaciones:

- Volumen, viscosidad y $p H$. El volumen del ejaculado (ml) se midió en tubos falcón graduados. La viscosidad se determinó midiendo la elasticidad (o filancia) del semen, colocando $0.05 \mathrm{ml}$ en una lámina portaobjetos y midiendo la distancia $(\mathrm{cm})$ entre la base de la lámina y la ruptura de la gota de semen al levantarla con una pipeta. El pH fue determinado con tiras reactivas de color.

- Motilidad. La motilidad fue evaluada en forma subjetiva, depositando $50 \mu 1$ de semen en una lámina porta objetos, colocada en una placa térmica. La lámina fue observada en un microscopio Nikon a un aumento de 40X. Para cada muestra, se evaluaron 10 campos con un mínimo de 200 espermatozoides por campo. La motilidad fue expresada en porcentaje.
- Test de endosmosis. Se añadió $25 \mu 1 \mathrm{de}$ semen a un vial conteniendo $500 \mu \mathrm{l}$ de la solución endosmótica en una concentración de $50 \mathrm{mOsm} / \mathrm{kg}$. Se incubó a $37^{\circ} \mathrm{C}$ por 20 min y luego se colocó $10 \mu 1$ de la solución en una lámina portaobjetos. Se observaron 100 espermatozoides al microscopio bajo un aumento de $40 \mathrm{X}$ (Giuliano et al., 2002). El test de endosmosis o HOST test permite evaluar la integridad funcional de la membrana y se fundamenta en los cambios morfológicos en los flagelos de los espermatozoides, con dilatación y enrollamiento, considerándose como respuesta positiva (Jeyendran et al., 1984).

- Concentración. Se coloca $0.1 \mathrm{ml}$ de semen en un vial con $0.9 \mathrm{ml}$ de agua, se homogeniza y se coloca en una cámara de Neubauer. Los espermatozoides se cuentan en un microscopio a un aumento de 40X. Los datos fueron expresados en números de espermatozoideS x $10^{6}$.

- Vitalidad. Se colocaron $10 \mu 1$ de semen en un portaobjeto y $10 \mu \mathrm{l}$ de la tinción Eosina-Nigrosina. Se hace un frotis y la lectura se hace al microscopio con aumento de $40 \mathrm{X}$. Se considera como muertos a los espermatozoides que adquieren color (Evans et al., 1999). Se cuenta como mínimo a 100 espermatozoides y los datos se expresan en porcentaje.

- Anormalidades. Se identificaron los espermatozoides anormales con ayuda de un microscopio y a un aumento de 40X. Los espermatozoides anormales fueron clasificados según la localización de la anormalidad (cola o cabeza), o si presentaban la gota citoplasmática proximal y gota citoplasmática distal.

- Temperatura ambiental. Los datos de temperatura fueron obtenidos del Servicio Nacional de Meteorología e Hidrología del Perú (SENHAMI) y se presentan en promedios por estación. 


\section{Análisis Estadístico}

Se realizó estadística descriptiva para los valores de las variables en estudio. Además, Los resultados obtenidos fueron analizados mediante la prueba de «t» Student para muestras independientes utilizando el paquete estadístico SPSS Statistics 22 (IBM).

\section{Resultados y Discusión}

Las características seminales en las dos épocas del año se muestran en el Cuadro 1. En las características macroscópicas solo se encontró diferencia significativa para la viscosidad (filancia), con valores más elevados en verano $(5.9 \pm 3.4 \mathrm{~cm})$ respecto a la época de invierno $(3.7 \pm 2.0 \mathrm{~cm})(\mathrm{p}<0.05)$, lo cual podría ser explicada por posibles mecanismos de protección del plasma seminal sobre la calidad de los espermatozoides, según lo señala Troedsson et al. (2005).

Respecto a las características microscópicas (Cuadro 1), los valores de motilidad, concentración, porcentaje de espermatozoides vivos, espermatozoides normales, y gota citoplasmática proximal fueron mayores para la época de invierno, mientras que las anormalidades de cola y de gota citoplasmática distal se incrementaron en el verano. Hansen (2009) y Pérez-Crespo et al. (2008) señalan igualmente el efecto negativo del estrés de calor sobre las características espermáticas y que luego ocurre una recuperación en la época de menor temperatura. Estos resultados son contradictorios al trabajo de Huanca et al. (2011) quienes no encontraron diferencias entre estaciones. Es posible que la mejor alimentación recibida por los animales en estudio haya favorecido la ocurrencia de estas diferencias. Por otro lado, Giuliano et al. (2008) reportan que la concentración espermática disminuye en verano en comparación con el invierno.
El volumen de los eyaculados se encuentra dentro del rango reportado en la literatura (Quispe et al., 1987; Garnica et al., 1993; Bravo et al., 2000), en tanto que el pH 7.2 promedio fue similar a otros reportes, indicando que, a pesar de diferencias entre las condiciones de crianza de las alpacas, estas variables no se afectan en forma significativa (Garnica et al., 1993; Bravo et al., 1997).

El rango de motilidad en el presente estudio fue entre 15 y $90 \%$, valores superiores al 5-80\% encontrado por Juyena et al. (2013) y al $20-72 \%$ reportado por Huanca et al. (2011), habiéndose empleado en todos los casos la vagina artificial para la colección de semen. En forma similar, la concentración espermática fue superior en el presente estudio (21-656 x 10\% $/ \mathrm{ml})$ al rango de 82500 250 000/ml reportado por Bravo et al. (1997) y de $11.13-49.83 \times 10^{6} / \mathrm{ml}$ reportado por Huanca et al. (2011) en estudios que fueron realizados en la sierra peruana.

Bravo et al. (1997) encontraron entre el 58 y $83 \%$ de espermatozoides vivos en eyaculados de alpacas, los cuales caen dentro del rango de 18 a $95 \%$ con una media de $58.7 \pm 18.0$ en el presente estudio.

Es importante enfatizar que los valores estuvieron afectados por los eyaculados colectados en el verano. Los porcentajes de espermatozoides normales muestran un rango de 65 a 96\% (88.1 $\pm 7.0 \%)$ para el invierno y de 68 a $84 \%(82.6 \pm 7.2 \%)$ para el verano; valores que se encuentran dentro del rango de 71-84\% reportado por Bravo et al. (1997). Por otro lado, las anormalidades en la cabeza (3.1\%), anormalidades en la cola $(6.7 \%)$, presencia de gota citoplasmática proximal $(2.4 \%)$ y distal (3.0\%) fueron relativamente inferiores a los reportados por Bravo et al. (1997) de 6.7\% para anormalidades de cabeza, $12.3 \%$ para anormalidades de cola y $3.8 \%$ para gotas citoplasmáticas. El aumento del porcentaje de anormalidades de cola en el verano coincide con los resultados encontrados en la zona andina por Huanca et al. (2011). 
Cuadro 1. Variables macroscópicas y microscópicas del semen de alpaca en época de verano e invierno (promedio \pm desviación estándar)

\begin{tabular}{lccc}
\hline Variables & Verano & Invierno & Total \\
\hline Volumen (ml) & $1.9 \pm 0.8$ & $2.1 \pm 0.8$ & $2.0 \pm 0.8$ \\
Filancia (cm) & $5.9 \pm 3.4^{\mathrm{a}}$ & $3.7 \pm 2.0^{\mathrm{b}}$ & $4.8 \pm 3.0$ \\
$\mathrm{pH}$ & $7.01 \pm 0.29$ & $7.1 \pm 0.3$ & $7.1 \pm 0.3$ \\
Motilidad (\%) & $58.0 \pm 22.1^{\mathrm{a}}$ & $77.3 \pm 11.6^{\mathrm{b}}$ & $67.6 \pm 20.0$ \\
Concentración (x10 $/ \mathrm{ml})$ & $135.9 \pm 88.7^{\mathrm{a}}$ & $242.4 \pm 140.9^{\mathrm{b}}$ & $189.2 \pm 128.1$ \\
Espermatozoides vivos (\%) & $52.9 \pm 18.4^{\mathrm{a}}$ & $64.5 \pm 15.9^{\mathrm{b}}$ & $58.7 \pm 18.0$ \\
HOST+ (\%) & $56.15 \pm 15.8$ & $49.3 \pm 13.2$ & $52.7 \pm 14.8$ \\
Espermatozoides normales (\%) & $82.6 \pm 7.2^{\mathrm{a}}$ & $88.1 \pm 7.0^{\mathrm{b}}$ & $85.3 \pm 7.6$ \\
Anormalidades en cola (\%) & $7.55 \pm 4.8^{\mathrm{a}}$ & $4.7 \pm 4.2^{\mathrm{b}}$ & $6.1 \pm 4.7$ \\
Anormalidades en cabeza (\%) & $3.5 \pm 3.6$ & $2.8 \pm 4.2$ & $3.1 \pm 3.9$ \\
Gota citopl. proximal (\%) & $1.7 \pm 1.4^{\mathrm{a}}$ & $3.2 \pm 2.6^{\mathrm{b}}$ & $2.4 \pm 2.2$ \\
Gota citopl. distal (\%) & $4.8 \pm 5.1^{\mathrm{a}}$ & $1.2 \pm 1.4^{\mathrm{b}}$ & $3.0 \pm 4.1$ \\
& & & \\
\hline
\end{tabular}

${ }^{a, b}$ Superíndices diferentes indican diferencias significativas $(p<0.05)$

En la época de verano se observaron diferencias significativas entre machos $(\mathrm{p}<0.05)$ para las variables volumen, filancia y $\mathrm{pH}$, mientras en que la época de invierno de encontraron diferencias entre machos $(\mathrm{p}<0.05)$ para las variables volumen, $\mathrm{pH}$, motilidad, HOST y espermatozoides normales. Estas diferencias entre individuos coinciden con el estudio de Giuliano et al. (2008), que indican que las diferencias individuales pueden ser atribuidas a las características propias de los animales.

\section{Conclusiones}

- Las características seminales en alpacas criadas en la costa peruana presentan diferencias significativas $(\mathrm{p}<0.05)$ entre las épocas de verano e invierno para las variables concentración, motilidad, filancia, vivos/muertos, normales/anormales, anormalidades de cola y gotas citoplasmáticas.
- Los valores encontrados en el presente estudio para las variables seminales difieren de otros estudios, lo que puede ser atribuido a diferencias en la alimentación.

\section{Literatura Citada}

1. Bravo PW, Flores $U$, Garnica $J$, Ordonez C. 1997. Collection of semen and artificial insemination of alpacas. Theriogenology 47: 619-626. doi: 10.1016/S0093-691X(97)00020-4

2. Bravo PW, Skidmore JA, Zhao XX. 2000. Reproductive aspects and storage of semen in camelidae. Anim Reprod Sci 62: 173-193. doi: 10.1016/S0378-4320(00)00158-5

3. Evans G, Maxwell WMC, Portal JI. 1990. Inseminación artificial de ovejas y cabras. Zaragoza, España: Ed. Acribia. 204 p.

4. [FAO]. 1996. Manual de practicas de manejo de alpacas y llamas. Roma: FAO. $107 \mathrm{p}$. 
5. Fernandez-Baca S. 1991. Avances y perspectivas del conocimiento de los camelidos sudamericanos. Santiago de Chile: Oficina Regional de la FAO para América Latina y el Caribe. 429 p.

6. Garnica J, Achata R, Bravo PW. 1993. Physical and biochemical characteristics of alpaca semen. Anim Reprod Sci 32: 85-90. doi: 10.1016/03784320(93)90059-Z

7. Giuliano S, Director A, Gambarotta M, Trasorras V, Miragaya M. 2008. Collection method, season and individual variation on seminal characteristics in the 1lama (lama glama). Anim Reprod Sci 104: 359-369. doi: 10.1016/j.anireprosci. 2007.02.016

8. Giuliano $S$, Ferrari $M$, Spirito $S$, Campi S, Director A, Fernández $H$. 2002. Methodological advances on the hypoosmotic swelling test (HOS test) in llama (Lama glama) spermatozoa. Biocell 27: 149 (Abstr).

9. Hansen PJ. 2009. Effects of heat stress on mammalian reproduction. Philos Trans R Soc Lond B Biol Sci 364: 3341 3350. doi: 10.1098/rstb.2009.0131

10. Huanca T, Mamani H, Naveros L, Pacheco J, Condor N. 2011. Variacion individual y estacional de las caracteristicas seminales en la alpaca (vicugna pacos). Spermova 1(1): 98-100.

11. Huanca W, Gauly M. 2001. Conservación de semen refrigerado de llamas. Rev Inv Vet Perú 12: 460-464.

12. Jeyendran R, Van Der Ven H, PerezPelaez M, Crabo B, Zaneveld L. 1984. Develoment on an assay to assess the functional integrity of the human sperm membrane and its relationship to the other sêmen characteristics. J Reprod Fert 70: 219-228.

13. Juyena NS, Vencato J, Pasini G, Vazzana I, Stelletta C. 2013. Alpaca semen quality in relation to different diets. Reprod Fertil Dev 25: 683-690. doi: 10.1071/RD12050

14. Pérez-Crespo M, Pintado B, Gutiérrez-Adán A. 2008. Scrotal heat stress effects on sperm viability, sperm DNA integrity, and the offspring sex ratio in mice. Mol Reprod Dev 75: 40-47. doi: 10.1002/mrd.20759

15. Quispe F. 1987. Evaluación de las características físicas del semen de la alpaca durante la época del empadre. Tesis de Bachiller. Puno, Perú: Univ. Nacional del Altiplano. $70 \mathrm{p}$.

16. Sumar J, Leyva C. 1981. Colección de semen mediante vagina artificial en la alpaca (Lama pacos). En: IV Convención Internacional sobre Camélidos Sudamericanos. Punta Arenas, Chile.

17. Troedsson MHT, Desvousges A, Alghamdi AS, Dahms B, Dow CA, Hayna J, Valesco R, et al. 2005. Components in seminal plasma regulating sperm transport and elimination. Anim Reprod Sci 89: 171-186. doi: 10.1016/ j.anireprosci.2005.07.005

18. Ungerfeld R. 2002. Termorregulacion testicular. En: Ungerfeld R (ed). Reproducción en los animales domésticos. Montevideo: Ed Melibea. p 114-118. 